PROTEIN SYNTHESIS

\title{
An expressive couple
}

Organisms must precisely regulate protein synthesis to tailor their response to environmental fluctuations. This regulation is complex and involves tight control at many levels, including transcription, mRNA decay and translation initiation. Harel-Sharvit et al. now find that the heterodimeric RNA polymerase II (RNAPII) subunits Rpb4 and $\mathrm{Rpb} 7$ (Rpb4-Rpb7) regulate translation initiation, demonstrating a novel link between transcription and translation in eukaryotes.

mRNAs shuttle between polysomes (sites of active translation) and processing bodies (PBs), the latter being complexes in which non-translating mRNAs accumulate and may be degraded. It is increasingly evident that translation and mRNA decay are intimately coupled and coordinately regulated. Transcription has also been linked to mRNA decay, as the co-transcriptional association between $\mathrm{Rpb} 4-\mathrm{Rpb} 7$ and mRNA regulates two major mRNA decay pathways.
Harel-Sharvit et al. found that Rpb4-Rpb7 interacts with components of translation initiator factor 3 and so they asked whether this might be important for translation initiation. They showed that the yeast mutants rpb7-26 (which exhibits normal transcription and mRNA degradation rates) and $r p b 4 \Delta$ are hypersensitive to translation inhibitors and to modulations in translation. Comparisons of protein synthesis between wild-type, rpb7-26 and $r p b 4 \Delta$ cells revealed that mutant cells consistently exhibited slower rates of protein synthesis, a lower proportion of polysomes and accumulation of PBs. In response to starvation, mRNAs are excluded from polysomes and accumulate in PBs. The reversal of this process that is normally seen upon re-feeding of wild-type cells was disrupted in rpb7-26 cells. Further experiments showed that, in mutant cells, dissociation of mRNAs from PBs and assembly of mRNAs with polysomes were impaired. Finally, the authors found that cells expressing RNAPII mutants that are unable to bind Rpb4-Rpb7, or that express an Rpb4 mutant that localizes to the cytoplasm, have defects in translation, showing that co-transcriptional association of Rpb4-Rpb7 with mRNA is important for translation in addition to its importance for mRNA export and decay.

These investigations have revealed a new link between transcription and translation that is mediated by Rpb4-Rpb7. Considering that Rpb4-Rpb7 regulates mRNA synthesis, export, translation and decay, the authors have named these proteins 'mRNA coordinators', as they are key for several regulatory steps that the mRNA undergoes, including its translation.

Mhairi Skinner, Consulting Editor, $\mathrm{NCl}-\mathrm{Nature}$ Pathway Interaction Database

ORIGINAL RESEARCH PAPER Harel-Sharvit, L. et al. RNA polymerase Il subunits link transcription and mRNA decay to translation. Cell 143, 552-563 (2010)

FURTHER READING Jackson, R. J., Hellen, C. U. T. \& Pestova, T. V. The mechanism of eukaryotic translation initiation and principles of its regulation. Nature Rev. Mol. Cell Biol.11, 113-127 (2010)

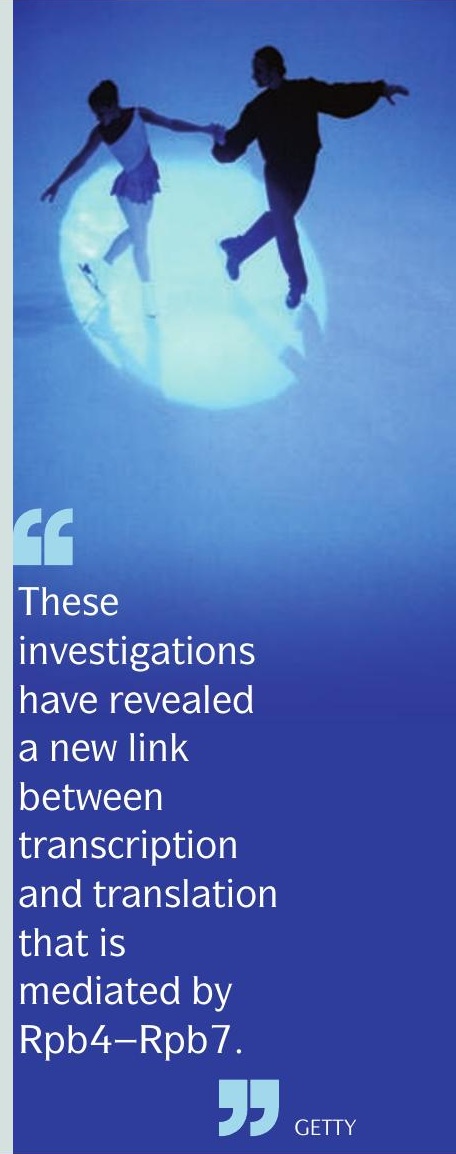

